\title{
Jubilación. ¿Existen actividades que faciliten la transición?
}

\author{
Martin Sanchez-Gomez \\ sanchgom@uji.es \\ Irene Belloch Tello \\ al362827@uji.es \\ Maria del Carme Ortiz Aguilar \\ al362801@uji.es \\ Paula González Fernández \\ al362844@uji.es \\ Edgar Breso Esteve \\ breso@uji.es
}

\section{Resumen}

Son diversas las investigaciones que han estudiado las diferentes etapas que hay en la jubilación y los estados de ánimo asociados a estas, frecuentemente percibidos de forma negativa. Sin embargo, pese a haberse descubierto la utilidad de mantenerse activo, tanto física como mentalmente, apenas existen datos acerca de cómo se sienten las personas según el tipo de actividad que realizan tras jubilarse. El objetivo del presente trabajo es conocer si existe relación entre la satisfacción de las personas jubiladas y el hecho de realizar actividades cognitivas en esta etapa. La muestra estuvo compuesta por 142 participantes (47,89\% mujeres y $52,11 \%$ hombres), con una edad media de 69,82 años. Para evaluar la satisfacción se tomaron dos medidas: una de satisfacción con la vida, medida con la Escala de Satisfacción con la Vida (SWLS), y otra de satisfacción con la jubilación, medida con un cuestionario de diseño propio formado por nueve ítems. Los participantes fueron divididos en tres grupos en función del tipo de actividad principal realizada tras la jubilación: acudir a centros de día, cursar estudios universitarios o no realizar ninguna actividad cognitiva. Para conocer las diferencias entre grupos se realizaron pruebas ANOVA. Los resultados indican que no se encuentran diferencias significativas ni en satisfacción con la vida ni en satisfacción con la jubilación entre los diferentes grupos. En futuras investigaciones sería conveniente ampliar la muestra y contemplar distintos tipos de actividades que puedan realizar las personas jubiladas más allá de las elegidas en este estudio.

Palabras clave: jubilación, calidad de vida, vejez, satisfacción, retiro. 


\begin{abstract}
Several studies have studied the different stages of retirement and the moods associated with them, however, there is hardly any information about how people feel depending on the type of activity they perform after retirement. The objective of this paper is to know if there is a relationship between the satisfaction of retired people and the fact of performing cognitive activities during this stage. The sample consisted of 142 participants $(47.89 \%$ women and $52.11 \%$ men), with an average age of 69.82 years. To evaluate satisfaction, two measures were taken: one of life satisfaction, measured with the Life Satisfaction Scale (SWLS), and another of satisfaction with retirement, measured with a self-designed questionnaire consisting of nine items. The participants were divided into three groups according to the type of main activity carried out after retirement: going to day centers, attending university studies, or not doing any cognitive activity. To know the differences between groups, ANOVA tests were performed. The results show no significant differences in life satisfaction and satisfaction with retirement among different groups. In future investigations it would be convenient to expand the sample and contemplate different types of activities that retired people can carry out beyond those chosen in this study.
\end{abstract}

Keywords: retirement, quality of life, old age, satisfaction, cognitive activity.

\title{
Introducción
}

En la actualidad, las sociedades y las urbes en las que vive la mayoría de la población se caracterizan por el ritmo frenético que las invade. La vida laboral ha ido cobrando año tras año mayor relavancia y tener que atender múltiples tareas se ha convertido en algo muy frecuente (Mazzetti, Schaufeli, Guglielmi y Depolo 2016). No obstante, alrededor de los 65 años se produce un cambio drástico con este modo de vida ocasionado por la llegada de la jubilación.

La jubilación es un evento vital clave en el contexto actual y se entiende como un derecho establecido y reglado por la seguridad social de cada país. La aceptación del fin del rol laboral no es igual para todas las personas. En ciertos casos esta nueva situación se puede convertir en algo traumático dependiendo de diversos factores, por lo que la aceptación de este nuevo rol es determinante para que la satisfacción con la vida en la etapa de jubilación sea adecuada (Cándido Corral, Díaz Castro, Duque Aparicio, Estévez Ortega y Gamero Larios 2016). El estado de salud físico y psicológico, el estatus socioeconómico o la personalidad son algunos factores relevantes que influyen en las experiencias que se producen al llegar a la jubilación (Aponte Daza 2015; Maurer y Chapman 2018).

El gerontólogo Robert Atchley (1976) propuso un modelo sobre la jubilación que consta de seis fases: prejubilación, jubilación, desencanto, reorientación, estabilidad y finalización. En la primera etapa, generalmente, la persona genera expectativas, tanto positivas como negativas. En la segunda, Atchley, concretó tres patrones de conducta diferentes: luna de miel, actividad continuada y descanso. Se habla de luna de miel cuando la persona percibe la jubilación como unas vacaciones indefinidas en las que realiza todo tipo de tareas que había dejado pendientes. Por otra parte, la actividad continuada ocurre cuando se reemplaza la actividad laboral por otras, principalmente 
de ocio. Finalmente, el descanso consiste en una disminución del nivel de actividad; este patrón, frecuentemente, se observa en personas que han trabajado mucho. En la fase de desencanto, normalmente, la persona experimenta sentimientos de tristeza, incertidumbre y decepción, especialmente si se generaron expectativas demasiado altas. La cuarta fase, reorientación, se considera una continuación de la anterior etapa, en la cual la persona reevalúa la situación de forma más realista. En la fase de estabilidad, la persona se adapta completamente a la jubilación mediante unas rutinas diarias. La última fase, finalización, la persona presenta un ajuste emocional y conductual al rol de jubilado. En un estudio realizado con una muestra de personas españolas recién jubiladas, se identificaron las etapas propuestas por Atchley, aunque no de forma ordenada ni en su totalidad (Aymerich Andreu, Planes Pedra y Gras Pérez 2010).

La investigación se ha centrado en conocer los cambios psicológicos asociados a esta etapa. En el estudio de Rodríguez Feijóo (2007) se investigaron las actitudes hacia la jubilación de una muestra de sujetos en las etapas prejubilación y posjubilación; los resultados demostraron que el $51 \%$ de la muestra presentaba actitudes desfavorables; el $13 \%$, actitudes neutras y el $36 \%$, actitudes favorables. En un estudio más reciente (Cándido Corral, Díaz Castro, Duque Aparicio, Estévez Ortega y Gamero Larios 2016) se investigó si la jubilación afecta a la satisfacción con la vida y a la autoestima. Con una muestra de 26 sujetos (la mitad recién jubilados y la otra mitad jubilados hace más de 3 años), los resultados mostraron que los jubilados recientes tienen menor satisfacción con la vida y menor autoestima que las personas que llevan más de 3 años jubiladas.

Según Moragas y Linz (1991), el trabajo y la ancianidad no son compatibles. El trabajo supone productividad y exigencias físicas, psíquicas y sociales; sin embargo, la ancianidad implica lo contrario, además del abandono de las obligaciones y las responsabilidades laborales. En la línea opuesta encontramos un estudio longitudinal en el se que analizó la relación entre jubilación y salud en una muestra de más de 12000 personas de entre 51 y 61 años. Los resultados de esta investigación demostraron que continuar realizando una actividad laboral moderada después de la jubilación predice una mejor salud física y psicológica en comparación con aquellos que no la realizan (Zhan, Wang, Liu y Shultz 2009). De la misma forma, estudios más recientes han demostrado que el mantenimiento de la actividad cognitiva durante los primeros años de jubilación está relacionado con una transición más adecuada (Vercambre, Okereke, Kawachi, Grodstein y Kang 2016) y con un mayor nivel de satisfacción en la calidad de vida general (Bielinska, Majkowicz, Waz y Bielinska-Waz 2018).

Cómo afrontan las personas el fin de su vida laboral para dar paso a esta nueva etapa ha sido estudiado en multitud de investigaciones. Sin embargo, son pocos los que han puesto el foco en descubrir qué actividades tienen mayor beneficio sobre la satisfacción personal. Por todo esto, el primer objetivo del presente trabajo es conocer si existen diferencias en la satisfacción con la vida entre personas jubiladas y aquellas que están a punto de serlo. Mientras que el segundo objetivo es conocer si existen diferencias en la satisfacción con la vida y la jubilación entre aquellos que realizan actividades cognitivas y los que no. En relación con el marco teórico, se hipotetiza que los niveles de satisfacción serán más altos en jubilados y que las personas que realizan actividades de tipo cognitivo tras la jubilación, como acudir a un centro de día o cursar estudios universitarios, mostrarán niveles más altos de satisfacción que aquellas personas que no desempeñan ningún tipo de actividad mental. 


\section{Método}

\section{Muestra}

El presente estudio sigue un diseño transversal. Para él se consiguió una muestra de 142 sujetos $(47,89 \%$ mujeres y $52,11 \%$ hombres), con una edad media de 69,82 años. Del total, 15 no estaban jubilados; ( $X \square=59,14$ años) mientras que los 127 restantes $(48,82 \%$ mujeres y $51,18 \%$ hombres) son jubilados con una edad media de 70,99 años. De los 127 jubilados: 43 cursan estudios en la Universidad Complutense de Madrid (51,16 \% mujeres y 48,84 \% de hombres; de edad 67,58 años), 25 acuden a centros de día para mayores donde realizaban diferentes actividades mentales (56\% mujeres y $44 \%$ hombres; media de edad 72,04 años) y $59(42,37 \%$ mujeres y $57,63 \%$ hombres, media de edad 72,85 años) declararon no realizar actividades de tipo cognitivo.

\section{Materiales}

Para llevar a cabo el estudio, se utilizó la Escala de Satisfacción con la Vida (SWLS) desarrollada por Diener, Emmons, Larsen y Griffin (1985). Se empleó la versión en español facilitada por el autor en su web y su valor alfa de Cronbach fue 0,82. Esta escala se compone de cinco ítems como «En la mayoría de los casos, mi vida está cerca de mi ideal», cuya respuesta está dada por una escala Likert que va de 1 (muy en desacuerdo) a 7 (muy de acuerdo).

Además, esta medida se complementó con un cuestionario de diseño propio sobre satisfacción con la jubilación formado por nueve ítems (véase el anexo). Las respuestas se midieron en base a una escala Likert de 1 a 10, siendo 1 totalmente en desacuerdo y 10 totalmente de acuerdo. El valor alfa de Cronbach fue 0,91.

\section{Procedimiento}

El cuestionario se realizó a pie de calle de manera voluntaria, en papel y de forma presencial, por tanto nos encontramos ante un muestreo por conveniencia (López 2004). La muestra se recogió en dos ciudades diferentes. Por una parte, los residentes en Castellón forman en gran medida la línea base, el grupo de centro de día y el grupo sin actividad cognitiva. Por la otra parte, los residentes en Madrid forman el resto de la línea base y el grupo de jubilados estudiantes pertenecientes a la Universidad Complutense de Madrid. Los participantes declararon su consentimiento para participar en la investigación y se les explicó que los datos iban a ser utilizados de manera anónima y confidencial. Todos ellos estuvieron siempre bajo la supervisión de varios investigadores que se ocuparon de que las cuestiones fueran entendidas y respondidas correctamente.

Una vez administrados los cuestionarios, la escala de satisfacción con la vida fue recodificada sobre 10 puntos para facilitar la comparación de los dos cuestionarios. En los siguientes apartados los resultados se presentan como valores promedio totales y no de forma específica para cada ítem.

\section{Análisis de datos}

Obtenidas las respuestas, se procedió a almacenar los datos en la base estadística y posteriormente a su análisis mediante el programa estadístico SPSS (IBM) en su 
versión 24.0. Efectuamos un análisis de fiabilidad para conocer la consistencia interna mediante el coeficiente alfa de Cronbach; la diferencia de medias mediante la $t$ de Student para muestras independientes y mediante ANOVA cuando hubo tres variables implicadas.

\section{Resultados}

Análisis descriptivos en función del tipo de actividad

En la tabla 1 se comparan las puntuaciones medias entre los grupos de participantes en función del tipo de actividad que realizan. Los 59 jubilados que declararon no realizar actividades cognitivas muestran una puntuación media de 7,38 en el cuestionario de satisfacción con la vida y 7,41 en el de satisfacción con la jubilación. Los 25 jubilados que acuden a un centro de día tienen una puntuación media de 7,62 en el cuestionario de satisfacción con la vida y 7,68 en el de satisfacción con la jubilación. Las personas jubiladas que cursan estudios universitarios obtienen una puntuación de 7,57 en satisfacción con la vida y 7,90 en satisfacción con la jubilación. Por su parte, el grupo de personas no jubiladas obtienen una puntuación media de 6,75 puntos en el cuestionario de satisfacción con la vida.

Tabla 1

Puntuaciones medias en función del tipo de actividad

\begin{tabular}{|c|c|c|c|c|c|c|c|c|}
\hline & \multicolumn{2}{|c|}{$\begin{array}{l}\text { Jubilados sin } \\
\text { actividades } \\
\text { cognitivas }\end{array}$} & \multicolumn{2}{|c|}{$\begin{array}{l}\text { Jubilados que } \\
\text { acuden a centros } \\
\text { de día }\end{array}$} & \multicolumn{2}{|c|}{$\begin{array}{l}\text { Jubilados que } \\
\text { cursan estudios } \\
\text { universitarios }\end{array}$} & \multicolumn{2}{|c|}{$\begin{array}{l}\text { Personas no } \\
\text { jubiladas }\end{array}$} \\
\hline & $M$ & DT & $M$ & DT & $M$ & DT & $M$ & DT \\
\hline $\begin{array}{l}\text { Satisfacción } \\
\text { con la vida }\end{array}$ & 7,38 & 1,59 & 7,62 & 1,27 & 7,57 & 1,46 & 6,75 & 1,22 \\
\hline $\begin{array}{l}\text { Satisfacción } \\
\text { con la } \\
\text { jubilación }\end{array}$ & 7,41 & 1,45 & 7,68 & 1,36 & 7,90 & 1,54 & - & - \\
\hline
\end{tabular}

Análisis diferenciales en función del tipo de actividad

La tabla 2 muestra los resultados en la prueba $t$ de Student relacionada con la satisfacción con la vida en función del estado laboral (jubilados frente a trabajadores en activo). Según se puede apreciar, al nivel de confianza del $95 \%$, los datos revelan diferencias significativas entre grupos $(t=1,18, p<0,04)$, siendo el tamaño del efecto moderado $(\mathrm{d}=0,51)$. Es el grupo de trabajadores en activo el que presenta puntuaciones menores en satisfacción. 
Tabla 2

Análisis diferenciales en función del estado laboral

\begin{tabular}{lllllll}
\hline & $\begin{array}{l}\text { Jubilados } \\
(n=127)\end{array}$ & \multicolumn{2}{l}{$\begin{array}{l}\text { En activo } \\
(n=15)\end{array}$} & & \\
\cline { 2 - 6 } & $M$ & DT & $M$ & DT & $t$ & $p$ \\
\hline Satisfacción con la vida & 7,47 & 1,09 & 6,75 & 1,22 & 1,18 &, 04 \\
\hline
\end{tabular}

La prueba t de Student relativa a la actividad mental compara las puntuaciones medias entre el grupo de participantes que declararon realizar actividades cognitivas $(N=68)$ y aquellos que indicaron no realizarlas $(\mathrm{N}=59)$. Los análisis no han encontrado diferencias significativas entre grupos en ninguna de las dos medidas: satisfacción con la vida $(\mathrm{t}=-0,29, \mathrm{p}=0,43)$ y satisfacción con la jubilación $(\mathrm{t}=-1,22, \mathrm{p}=0,33)$.

En el análisis ANOVA correspondiente al tipo de actividad (acudir a un centro de día para mayores, cursar estudios universitarios y no realizar ninguna actividad cognitiva) tampoco se encuentran diferencias estadísticamente significativas en las puntuaciones medias de los tres grupos, ni en satisfacción con la vida $(F=1,32, p=0,15)$ ni en satisfacción con la jubilación ( $\mathrm{F}=1,15, \mathrm{p}=0,21)$.

\section{Discusión y conclusiones}

El primer objetivo de esta investigación ha sido estudiar la diferencia en la satisfacción con la vida entre personas laboralmente activas y jubiladas. Si se comparan los valores medios de satisfacción con la vida durante la fase laboral activa y los valores medios durante la jubilación, se observan diferencias significativas. Por lo tanto, la jubilación parece ser un evento vital clave implicado en los niveles de satisfacción de las personas. Estos resultados van en la línea de lo hallado en el estudio de Rodríguez Feijóo (2007) en el que el $59 \%$ de personas que se encontraban activas laboralmente tenían una actitud desfavorable, mientras que el porcentaje de actitud desfavorable entre las personas jubiladas era de un $43 \%$. Según Feijóo, la visión más optimista de este proceso de las personas jubiladas podría deberse a la mejora económica, ya que no tenían que gastarse dinero en asuntos relacionados con su trabajo (como transporte, comidas en restaurantes, etc.) y, además, no debían gastar dinero en la manutención de los hijos, puesto que ya se encontraban independizados. Los resultados aquí hallados también van en línea con el modelo de jubilación de Atchley (1976), ya que en las dos primeras fases, prejubilación y jubilación, se generan experiencias positivas como consecuencia del descanso y la realización de nuevas actividades.

El segundo objetivo ha sido estudiar las diferencias en la satisfacción con la vida y la jubilación en función de realizar, o no, actividades cognitivas en esta etapa. Los resultados obtenidos muestran que no existen diferencias significativas entre las personas que realizan actividades de tipo cognitivo, como acudir a un centro de día o cursar estudios universitarios, y las que no desempeñan actividades de tipo mental. Según estudios previos (Zhan, Wang, Liu y Shultz 2009; Vercambre, Okereke, Kawachi, Grodstein y Kang 2016), la realización de actividades durante la jubilación ha demostrado tener beneficios en cuanto a salud física y psicológica. En relación con la investigación aquí presentada, podemos resaltar que, pese a no encontrar diferencias significativas, el grupo de jubilados que realiza actividades mentales presenta una puntuación media superior respecto al otro grupo. 
En conclusión, podemos afirmar que existen diferencias significativas en la satisfacción con la vida entre personas jubiladas y aquellas que están a punto de serlo, siendo esta mayor en el grupo de jubilados. Por su parte, podemos decir que no hay diferencias significativas en la satisfacción con la vida y la jubilación entre los grupos que llevan a cabo actividad cognitiva y los que no desempeñan ningún tipo de actividad mental. Por tanto, parece que realizar este tipo de actividades en la jubilación no tiene relación con los niveles de bienestar.

En cuanto a los aspectos que se pueden mejorar en futuras investigaciones, es importante destacar que, a la hora de hablar de un concepto multideterminado como la satisfacción, se podría haber contemplado la importancia de otros aspectos tales como la salud física, el contacto social o el estatus socioeconómico, fundamentales en la vida de una persona. Pese a ello, se espera que esta investigación aporte luz al estudio de la jubilación y las formas de mejorar la satisfacción en esta etapa vital.

\section{Referencias bibliográficas}

Aponte Daza, Vaneska Cindy. 2015. «Calidad de vida en la tercera edad». Ajayu Órgano de Difusión Científica del Departamento de Psicología UCBSP, 13(2): 152-182.

Atchley, Robert C. 1976. The sociology of retirement. New York: John Wiley.

Aymerich Andreu, María, Montserrat Planes Pedra y María Eugenia Gras Pérez. 2010. "La adaptación a la jubilación y sus fases: Afectación de los niveles de satisfacción y duración del proceso adaptativo». Anales de Psicología, 26(1): 80-88.

Bielinska, Agnieszka, Mikolaj Majkowicz, Piotr Waz y Dorota Bielinska-Waz. 2018. "Overall quality of life and general health-changes related to the retirement threshold». eTELEMED 2018, 2: 9.

Cándido Corral, Andrés, Cristina Díaz Castro, Carolina Duque Aparicio, Eva Estévez Ortega y Jerónimo Gamero Larios. 2016. «Influencia de la jubilación en la satisfacción con la vida y la autoestima». ReiDoCrea, 5(2): 21-26.

Diener, Ed D., Robert A. Emmons, Randy J. Larsen y Sharon Griffin. 1985. «The satisfaction with life scale». Journal of personality assessment, 49(1): 71-75.

López, Pedro Luis. 2004. «Población muestra y muestreo». Punto cero, 9(8): 69-74.

Maurer, Todd J. y Elizabeth F. Chapman. 2018. «Relationship of proactive personality with life satisfaction during late career and early retirement». Journal of Career Development, 45(4): 345-360.

Mazzetti, Greta, Wilmar B. Schaufeli, Dina Guglielmi y Marco Depolo. 2016. «Overwork climate scale: psychometric properties and relationships with working hard». Journal of Managerial Psychology, 31(4): 880-896.

Moragas, Ricardo M. y Juan J. Linz. 1991. Gerontología social: envejecimiento y calidad de vida. Barcelona: Herder.

Rodríguez Feijóo, Nélida. 2007. «Actitudes hacia la jubilación». Interdisciplinaria, 24( 1): 5-42.

Vercambre, Marie N., Olivia I. Okereke, Ichiro Kawachi, Francine Grodstein y Jae H. Kang. 2016. "Self-reported change in quality of life with retirement and later cognitive decline: prospective data from the Nurses' Health Study». Journal of Alzheimer's Disease, 52(3): 887-898.

Zhan, Yujie, Mo Wang, Songqi Liu y Kenneth S. Shultz. 2009. «Bridge employment and retirees' health: A longitudinal investigation». Journal of occupational health psychology, 14(4): 374-389. 


\section{Anexo}

Cuestionario de diseño propio, el cual consta de nueve preguntas relacionadas con la satisfacción respecto a la jubilación.

Por favor, conteste a este cuestionario con la mayor sinceridad posible, ya que los datos serán tratados de forma completamente anónima. Deberá contestar a cada ítem indicando con un círculo una de las opciones de respuesta, indicando con un número su edad actual y de jubilación y con un valor del 1 al 10 los ítems numerados, según el tipo de pregunta y las opciones de respuesta.

1. Mi vida de jubilado se acerca a mi ideal en la mayoría de los sentidos.

2. Las condiciones de mi vida como jubilado son excelentes.

3. Tras mi jubilación, me encuentro satisfecho con mi vida.

4. Desde que me he jubilado, estoy logrando mis objetivos.

5. Mi vida era mejor antes de la jubilación.

6. La jubilación ha resultado mejor de lo que me esperaba.

7. Me siento estresado desde que me he jubilado.

8. Realizo tareas que me satisfacen tras mi jubilación.

9. Me siento feliz desde mi jubilación. 